\title{
Neutrophil to lymphocyte ratio as an indicative of diabetic control level in type 2 diabetes mellitus
}

\author{
Tuba T Duman, Gulali Aktas, Burcin M Atak, Mehmet Z Kocak, Edip Erkus, Haluk Savli
}

Abant Izzet Baysal University Hospital, Department of Internal Medicine, Bolu, Turkey.

\begin{abstract}
Background: Type 2 diabetes mellitus is associated with chronic low grade inflammation. One of the novel inflammatory markers is hemogram derived neutrophil to lymphocyte ratio (NLR).

Objective: We aimed to compare NLR levels of diabetic subjects and healthy controls and to observe possible correlation between NLR and HbA1c.

Methods: Medical data of type 2 diabetic subjects admitted to out-patient clinics of our institution between April to July in 2017 were obtained from database and retrospectively analyzed. Control group was chosen from healthy subjects who visited our institution for a routine check-up. Anthropometric measures, laboratory data, including, HbA1c, NLR were recorded.

Results: Median NLR of the type 2 DM group 2.44 (1.9) was significantly elevated when compared to healthy controls (1.5 (0.9), $(\mathrm{p}<0.001)$. In addition, a Pearson's correlation test revealed that NLR was strongly correlated with age $(\mathrm{r}=0.26, \mathrm{p}=0.008)$, fasting plasma glucose $(r=0.38, \mathrm{p}<0.001)$, and HbA1c $(r=0.49, \mathrm{p}<0.001)$.

Conclusion: Elevated NLR in otherwise healthy subjects may be indicative of underlying impaired glucose metabolism and moreover, NLR should be used as a marker of diabetic control level in addition to HbA1c in type 2 diabetic subjects.

Keywords: Type 2 diabetes mellitus, HbA1c, neutrophil to lymphocyte ratio, inflammation.

DOI: https://dx.doi.org/10.4314/ahs.v19i1.35

Cite as: Duman TT, Aktas G, Atak BM, Kocak MZ, Erkus E, H S. Neutrophil to lymphocyte ratio as an indicative of diabetic control level in type 2 diabetes mellitus. Afri Health Sci. 2019;19(1). 1602-1606. bttps:/ / dx.doi.org/10.4314/abs.v19i1.35
\end{abstract}

\section{Introduction}

Type 2 diabetes mellitus (DM) is one of the most important diseases that poses enormous burden to healthcare systems worldwide. Plasma glycated hemoglobin (HbA1c) is helpful in establishing the control level of the disease in subjects with DM. Increased levels of HbA1c are associated with worse outcome in the course of the disorder ${ }^{1}$. Moreover, recent studies suggest that inflammatory pathways were activated in type $2 \mathrm{DM}^{2,3}$.

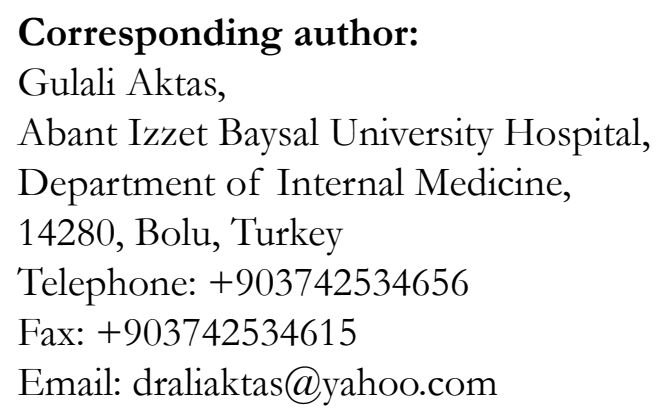

Novel inflammatory markers derived from standard blood count test are in demand nowadays. One of these derived novel inflammatory markers is neutrophil to lymphocyte ratio (NLR), which is got by simply division of neutrophil count by lymphocyte count in hemogram. It reflects high inflammatory burden of certain diseas$\mathrm{es}^{4}$. Authors found that NLR was significantly elevated in subjects with chronic obstructive pulmonary disease compared to the healthy population ${ }^{5}$. Latest studies in literature have found significant association between NLR and many conditions ${ }^{6-10}$.

The aim of present study was establishing NLR levels of diabetic subjects and comparing to those in healthy controls. We also aimed to find out possible correlation between NLR and HbA1c, and microvascular complications of type $2 \mathrm{DM}$.

\section{Methods}

Medical database of type 2 diabetic subjects who were

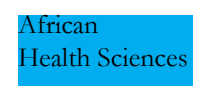

(C) 2019 Duman et al. Licensee African Health Sciences. This is an Open Access article distributed under the terms of the Creative commons Attribution License (https://creativecommons.org/licenses/BY/4.0), which permits unrestricted use, distribution, and reproduction in any medium, provided the original work is properly cited. 
admitted to out-patient clinics of our institution between April 2017 and July 2017, has been retrospectively analyzed, after approval of institutional board. Control subjects were enrolled from healthy subjects that applied to our clinics for a routine check-up. Exclusion criteria were as follows: active inflammation, infection, malignancy and pregnancy for women patients. Subjects younger than 18 years of age were also excluded. Age, gender, height, weight, and waist circumference of the participants were obtained from computerized database and recorded. A body mass index (BMI) was calculated by dividing of the weight in kilograms to the square of height in meters.

Venous blood samples obtained into sterile standard tubes containing constant amount of anticoagulant (K-EDTA). Laboratory tests have been held within several minutes after blood samples obtained. Serum urea, creatinine, fasting plasma glucose, total, HDL and LDL cholesterol, triglyceride and albumin were obtained from the same database and recorded. Urinary protein excretion, plasma HbA1c levels, and data about smoking or drinking habits, diabetic nephropathy, retinopathy and neuropathy were also obtained and recorded from patient file system. We also recorded white blood cell count (WBC), neutrophil count (neu), lymphocyte count (lym), hemoglobin (Hb), hematocrit (Htc) and platelet count (PLT). A NLR value was obtained by division of neu by lym.

Data were analyzed by SPSS software. (SPSS 15.0; IBM Inc., Chicago, IL, USA). Homogenous variables in study groups were expressed as mean \pm standard deviation and compared by independent samples t test, whereas, non-homogenous variables were expressed as median (Interquartile Range) and compared by Mann-Whitney U test. Comparison of categorical variables in study groups was conducted with Chi-square test. Correlation between study parameters was done with Pearson's correlation analyze test. A p value of $<0.05$ is considered as statistically significant.

\section{Results}

Study population was consisted of 110 subjects; 77 patients with type $2 \mathrm{DM}$ and 33 healthy controls. Mean age of the patients with type $2 \mathrm{DM}(58.6 \pm 10.9$ years $)$ was significantly higher than that of the control group (39.2 $\pm 12.4),(p<0.001) .37$ of 77 in type 2 DM group and 22 of 33 in control group were women. Gender was not statistically different in study groups $(p=0.07)$. Despite height was not statistically different in type $2 \mathrm{DM}$ group compared to control group $(\mathrm{p}=0.11)$, weight $(\mathrm{p}<0.001)$, BMI $(p<0.001)$ and waist circumference $(p<0.001)$ were significantly elevated in diabetics compared to healthy controls.

Only 3 of 33 subjects were smoking in the control group while 22 of 77 in type 2 DM group had smoking as a habit. The difference was reached the statistically significance level $(p=0.03)$. None of the controls and only 3 of the diabetics were drinking alcohol. The difference between groups was not significant $(\mathrm{p}=0.25)$.

Fasting plasma glucose of type 2 diabetic patients was significantly higher than control subjects $(p<0.001)$. Although $\mathrm{Hb}(p=0.83)$, Htc $(p=0.39)$, and PLT $(p=0.63)$ were similar in diabetics and controls, NLR of the type 2 DM group (2.44 [1.9]) was significantly elevated when compared to healthy controls $(1.5[0.9]),(p<0.001)$. On the other hand, WBC of diabetic population was significantly increased compared to control subjects $(p<0.001)$. 
Table 1. General characteristics and laboratory data of the study population

\begin{tabular}{|c|c|c|c|c|}
\hline & & Type 2 DM group & Control Group & $\mathbf{p}$ \\
\hline \multirow{3}{*}{ Gender } & Men (n) & 40 & 11 & \multirow{2}{*}{0.07} \\
\hline & Women $(\mathrm{n})$ & 37 & 22 & \\
\hline & & \multicolumn{2}{|l|}{ Mean $\pm S D$} & \\
\hline \multicolumn{2}{|c|}{ Age (years) } & $58.6 \pm 10.9$ & $39.2 \pm 12.4$ & $<0.001$ \\
\hline \multicolumn{2}{|c|}{ Height $(\mathrm{m})$} & $1.63 \pm 0.09$ & $1.66 \pm 0.08$ & 0.11 \\
\hline \multicolumn{2}{|c|}{ Weight $(\mathrm{kg})$} & $82 \pm 13$ & $70 \pm 9$ & $<0.001$ \\
\hline \multicolumn{2}{|c|}{ Waist circumference $(\mathrm{cm})$} & $106 \pm 12$ & $87 \pm 11$ & $<0.001$ \\
\hline \multicolumn{2}{|c|}{$\operatorname{BMI}\left(\mathrm{kg} / \mathrm{m}^{2}\right)$} & $30.9 \pm 5.5$ & $25.4 \pm 4.3$ & $<0.001$ \\
\hline \multicolumn{2}{|c|}{$\mathrm{Hb}(\mathrm{g} / \mathrm{dL})$} & $13.6 \pm 1.6$ & $13.6 \pm 1.8$ & 0.83 \\
\hline \multicolumn{2}{|c|}{ Htc $(\%)$} & $39.8 \pm 4.5$ & $40.7 \pm 5.1$ & 0.39 \\
\hline & & \multicolumn{2}{|l|}{ Median $(I Q R)$} & \\
\hline \multicolumn{2}{|c|}{ Fasting glucose $(\mathrm{mg} / \mathrm{dL})$} & $160(69)$ & $93(10)$ & $<0.001$ \\
\hline \multicolumn{2}{|c|}{$\mathrm{WBC}\left(\mathrm{k} / \mathrm{mm}^{3}\right)$} & $7.5(2.4)$ & $6.2(2.2)$ & $<0.001$ \\
\hline \multicolumn{2}{|l|}{ NLR } & $2.44(1.9)$ & $1.5(0.9)$ & $<0.001$ \\
\hline \multicolumn{2}{|c|}{$\operatorname{PLT}\left(\mathrm{k} / \mathrm{mm}^{3}\right)$} & $230(86)$ & $234(57)$ & 0.63 \\
\hline
\end{tabular}

BMI: body mass index, IQR: interquartile range

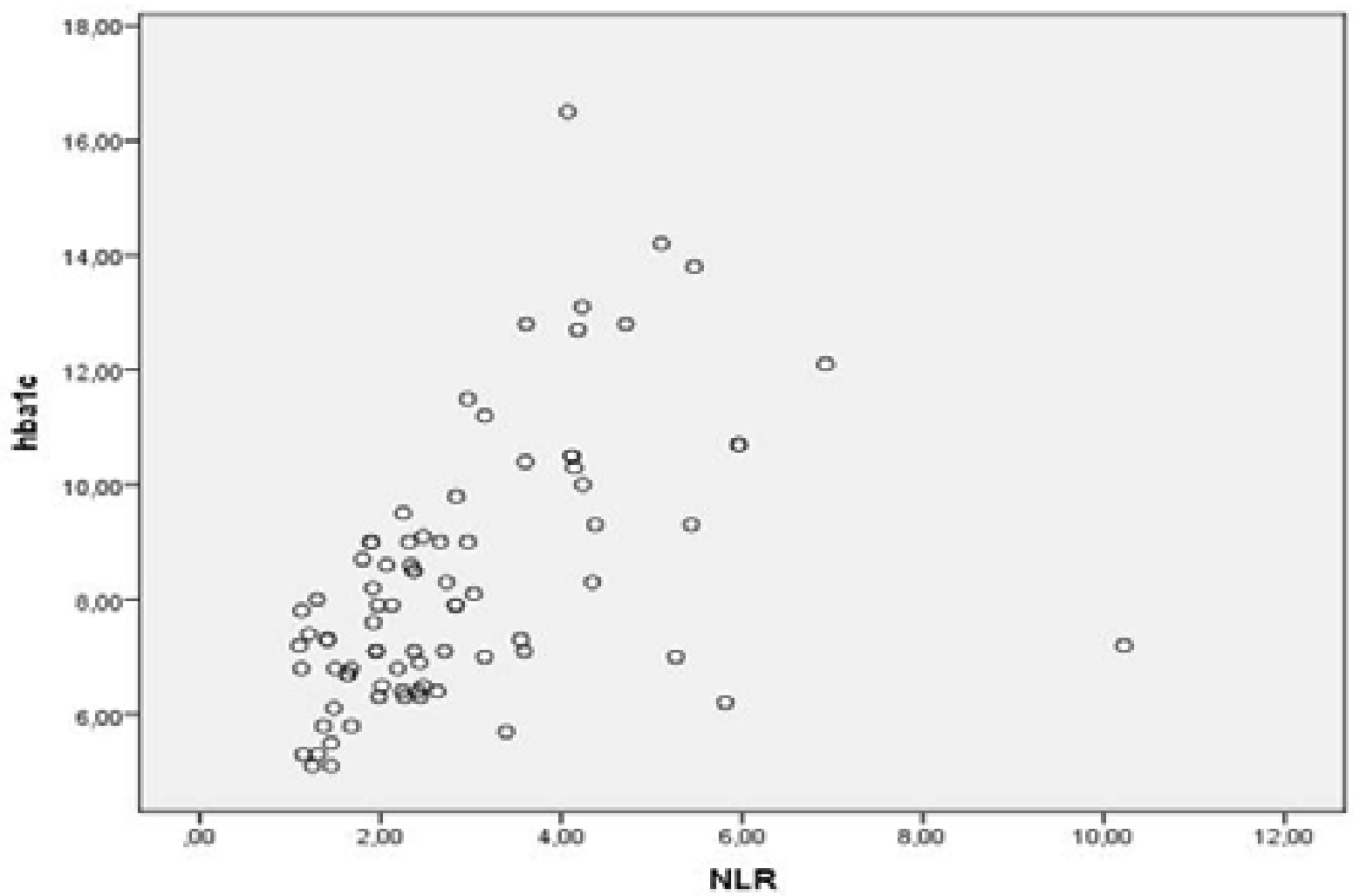

Figure 1. Correlation between HbA1c and NLR 
Table 1 shows general characteristics and laboratory data of the study groups. After adjustment of age difference between groups in regression analysis, NLR was still significantly different between Type $2 \mathrm{DM}$ and control groups (beta:-0.47, p<0.001, 95\% CI: $-0.73-0.26$ ).

A Pearson's correlation test revealed that NLR was strongly correlated with age $(r=0.25, p=0.008)$, fasting plasma glucose $(\mathrm{r}=0.35, \mathrm{p}<0.001)$, and HbA1c $(\mathrm{r}=0.51$, $\mathrm{p}<0.001)$. However, NLR was not correlated with BMI or with waist circumference. Figure 1 shows the correlation between HbA1c and NLR.

In sub-group analyze of diabetic patients, 34 had proteinuria of various levels and 43 had not. The NLR of type 2 diabetics with proteinuria was significantly higher than that of the diabetic patients without proteinuria $(p=0.008)$. NLR of diabetic patients with and without retinopathy was not statistically different $(p=0.91)$. Similarly, NLR levels of diabetic subjects with and without neuropathy were not significantly different $(p=0.17)$.

\section{Discussion}

Present study showed that NLR levels were increased in patients with type $2 \mathrm{DM}$. This increase probably showed the inflammatory burden of the disease. Moreover, our study also showed that NLR further increased as the HbA1c level worsened. Therefore, the results of present retrospective study arises the question whether NLR could be a novel marker of diabetic control.

In 2004, Ohshita et al showed increased WBC count in patients with impaired glucose tolerance ${ }^{11}$. Another study from China suggested their results by reporting an association between elevated WBC and diabetic complications $^{12}$. The increase in WBC mainly reflects the elevated neutrophil count in these studies. Similar to the literature, we found that WBC was higher in patients with type 2 DM than control subjects.

Association between inflammatory conditions and elevated NLR has been well-established ${ }^{13,14}$. Authors evenfound elevated NLR in another inflammatory condition, familial Mediterranean fever ${ }^{15}$. Increased NLR in type 2 DM should be explained by the association of the disease to chronic low grade inflammation ${ }^{2,16}$.

In a study in 2014, authors compared NLR levels of patients with well and poorly controlled diabetes mellitus, and found that NLR was higher in poorly controlled diabetics compared to well-controlled subjects ${ }^{17}$. Similar to our results in present study, they reported a positive correlation between NLR and HbA1c but not with $\mathrm{BMI}^{17}$. The distinction and an addition of present study is that we also figured out increased NLR of type 2 diabetics compared to healthy control subjects. To our knowledge, this is the first study reporting such a comparison between healthy and diabetic populations.

Underlying mechanisms of elevated NLR in type 2 DM need to be discussed. Neutrophils are the main branch of leukocytes in blood stream. Initially, and rapidly they respond to the inflammatory stimuli and neutrophil count increases in circulation. On the other hand, interleukin levels that increased in inflammatory conditions, cause lymphopenia ${ }^{18}$ and neutrophilia ${ }^{19}$, together causing elevated NLR.

Researchers reported that NLR was elevated by aging in healthy population ${ }^{20}$. Diabetic subjects were significantly older than controls in present study. However, we did not match the age of study groups for the sake of avoiding selection bias. Other limitations of our report are retrospective design and relatively small study population. They all make our results difficult to interpret. Nevertheless, we think that unique findings of present study are quite important to be enlisted in medical literature.

\section{Conclusion}

Elevated NLR in otherwise healthy subjects may be indicative of underlying impaired glucose metabolism and moreover, NLR should be used as a marker of diabetic control level in addition to $\mathrm{HbA1c}$ in type 2 diabetic subjects.

\section{Conflict of interest}

None to declare.

\section{Funding}

This work has not received any funds from any organizations.

\section{References}

1. Rohlfing CL, Wiedmeyer H-M, Little RR, England JD, Tennill A, Goldstein DE. Defining the relation- 
ship between plasma glucose and HbA1c. Diabetes care. 2002;25(2):275-278.

2. Pradhan AD, Manson JE, Rifai N, Buring JE, Ridker PM. C-reactive protein, interleukin 6, and risk of developing type 2 diabetes mellitus. JAMA. 2001;286(3):327334.

3. Stehouwer CD, Gall M-A, Twisk JW, Knudsen E, Emeis JJ, Parving H-H. Increased urinary albumin excretion, endothelial dysfunction, and chronic low-grade inflammation in type 2 diabetes. Diabetes. 2002;51(4):1157-1165.

4. Motomura T, Shirabe K, Mano Y, Muto J, Toshima T, Umemoto Y, et al. Neutrophil-lymphocyte ratio reflects hepatocellular carcinoma recurrence after liver transplantation via inflammatory microenvironment. Journal of Hepatology. 2013;58(1):58-64.

5. Paliogiannis P, Fois AG, Sotgia S, Mangoni AA, Zinellu E, Pirina $P$, et al. The neutrophil-to-lymphocyte ratio as a marker of chronic obstructive pulmonary disease and its exacerbations: A systematic review and meta-analysis. Eur J Clin Invest. 2018:e12984.

6. Bhat T, Teli S, Rijal J, Bhat H, Raza M, Khoueiry G, et al. Neutrophil to lymphocyte ratio and cardiovascular diseases: a review. Expert Review of Cardiovascular Therapy. 2013;11(1):55-59.

7. Tamhane UU, Aneja S, Montgomery D, Rogers E-K, Eagle KA, Gurm HS. Association between admission neutrophil to lymphocyte ratio and outcomes in patients with acute coronary syndrome. The American Journal of Cardiology. 2008;102(6):653-657.

8. Halazun K, Aldoori A, Malik H, Al-Mukhtar A, Prasad $\mathrm{K}$, Toogood G, et al. Elevated preoperative neutrophil to lymphocyte ratio predicts survival following hepatic resection for colorectal liver metastases. European Journal of Surgical Oncology (EJSO). 2008;34(1):55-60.

9. Walsh S, Cook E, Goulder F, Justin T, Keeling N. Neutrophil-lymphocyte ratio as a prognostic factor in colorectal cancer. Journal of Surgical Oncology. 2005;91(3):181-184. 10. Núñez J, Núñez E, Bodí V, Sanchis J, Miñana G, Mainar L, et al. Usefulness of the neutrophil to lymphocyte ratio in predicting long-term mortality in ST segment elevation myocardial infarction. The American Journal of Cardiology. 2008;101(6):747-752.
11. Ohshita K, Yamane K, Hanafusa M, Mori H, Mito $\mathrm{K}$, Okubo M, et al. Elevated white blood cell count in subjects with impaired glucose tolerance. Diabetes Care. 2004;27(2):491-496.

12. Tong PC, Lee K-F, So W-Y, Ng MH, Chan W-B, Lo $\mathrm{MK}$, et al. White blood cell count is associated with macro-and microvascular complications in Chinese patients with type 2 diabetes. Diabetes Care. 2004;27(1):216-222.

13. Moore M, Chua W, Charles K, Clarke S. Inflammation and cancer: causes and consequences. Clinical Pharmacology \& Therapeutics. 2010;87(4):504-508.

14. Shimada H, Takiguchi N, Kainuma O, Soda H, Ikeda A, Cho A, et al. High preoperative neutrophil-lymphocyte ratio predicts poor survival in patients with gastric cancer. Gastric Cancer. 2010;13(3):170-176.

15. Uslu AU, Deveci K, Korkmaz S, Aydin B, Senel S, Sancakdar E, et al. Is neutrophil/lymphocyte ratio associated with subclinical inflammation and amyloidosis in patients with familial Mediterranean fever? BioMed Research International. 2013;2013.

16. Thorand B, Löwel H, Schneider A, Kolb H, Meisinger C, Fröhlich M, et al. C-reactive protein as a predictor for incident diabetes mellitus amongmiddle-aged men: results from the MONICA Augsburg cohort study, 19841998. Archives of Internal Medicine. 2003;163(1):93-99.

17. Sefil F, Ulutas KT, Dokuyucu R, Sumbul AT, Yengil $\mathrm{E}$, Yagiz AE, et al. Investigation of neutrophil lymphocyte ratio and blood glucose regulation in patients with type 2 diabetes mellitus. Journal of International Medical Research. 2014;42(2):581-588.

18. Chung K-P, Chang H-T, Lo S-C, Chang L-Y, Lin S-Y, Cheng A, et al. Severe lymphopenia is associated with elevated plasma interleukin-15 levels and increased mortality during severe sepsis. Shock. 2015;43(6):569-575.

19. Fonseka TM, McIntyre RS, Soczynska JK, Kennedy SH. Novel investigational drugs targeting IL-6 signaling for the treatment of depression. Expert Opinion on Investigational Drugs. 2015;24(4):459-475.

20. Li J, Chen Q, Luo X, Hong J, Pan K, Lin X, et al. Neutrophil to Lymphocyte Ratio Positively Correlates to Age in Healthy Population. Journal of Clinical Laboratory Analysis. 2015;29(6):437-443. 